\title{
Coronary Revascularization Procedure
}

National Cancer Institute

\section{Source}

National Cancer Institute. Coronary Revascularization Procedure. NCI Thesaurus. Code C119202.

Any procedure that is undertaken to reestablished blood flow to the heart. 